\title{
in a Young Boy After Receiving a Diphtheria, Tetanus, and Acellular Pertussis Vaccine
}

\author{
Sarang S. Koushik, MD' \\ Vanny Le, MD² \\ Naum Shaparin, $\mathrm{MD}^{3}$ \\ Andrew Kaufman, $\mathrm{MD}^{4}$
}

\begin{abstract}
Background: Complex regional pain syndrome (CRPS) is a clinically diagnosed multifaceted condition comprising of physical complaints often with a psychological component. CRPS can result in devastating chronic pain and disability if not treated early and aggressively. Mostly seen in adults, CRPS is being described more frequently in children. There have been some rare reports of CRPS occurring after vaccine administration. This is a case report of a young boy who developed CRPS after receiving a routine vaccination.
\end{abstract}

Case

Presentation: Although CRPS has been reported after rubella and hepatitis B vaccine, this is a case of CRPS after a diphtheria, tetanus, and acellular pertussis vaccine. Delays in diagnosis may cause undue pain, extended lengths of treatment, requirements for more intense therapy, and less likelihood for complete functional restoration. Because there seems to be no common constituent to the vaccines described in this case, the resultant CRPS may be purely due to injection-site trauma.

Conclusion: We encourage physicians to be wary of the syndrome and its link to multiple vaccines in order to initiate prompt treatment.

Key words: Complex regional pain syndrome (CRPS), reflex sympathetic dystrophy (RSD), children, vaccination, diphtheria, tetanus, acellular pertussis (TDAP)

\section{BACKGROUND}

Complex regional pain syndrome (CRPS) is a clinically diagnosed multifaceted condition comprising of physical complaints often with a psychological component (1). CRPS can result in devastating chronic pain and disability if not treated early and aggressively (2). Mostly seen in adults, CRPS is being described more frequently in children $(3,4)$. There have been some rare reports of CRPS occurring after vaccine administration.

\section{CASE}

A 10-year-old boy with no significant medical history

\footnotetext{
From: 'Department of Anesthesiology Keck School of Medicine of University of Southern California, Los Angeles, CA; ${ }^{2}$ Riverside Medical Group, Clinton, NJ; ${ }^{3}$ Montefiore Medical Center of the Albert Einstein College of Medicine, Bronx, NY; ${ }^{4}$ Rutgers The State University of New Jersey, Newark, NJ 
presented to his pediatrician for a routine diphtheria, tetanus, and acellular pertussis (Tdap) booster and Varicella virus vaccine; the Tdap was given in the left deltoid and the varicella was given in the right deltoid.

The next day, the boy complained of pain in his left hand. He attended school but was unable to participate in gym. The patient returned to his pediatrician the following day and was found to have swelling on the thenar eminence with tenderness and inflammation in the second and third metacarpals of the left hand. There was no irritation at the injection site. The pediatrician immediately referred the patient to a pediatric orthopedist. The orthopedist noted mild left wrist and hand swelling with ecchymotic discoloration. There was tenderness with decreased range of motion. A radiograph of the hand was negative. When the symptoms began to worsen, the patient was referred to the pain medicine center.

He presented to the pain clinic less than one week from the inciting event. The symptoms progressed to include pain with any movement of the left hand, wrist, and elbow. His left arm was in full extension with his forearm pronated and his wrist in a neutral position. There was significant skin mottling. Allodynia was present over the palmar surface, hyperalgesia over the dorsum, and mild hypersensitivity over the forearm. There was considerable decreased range of motion with both supination and pronation of the arm as well as flexion and extension of the wrist. The patient was able to move his left shoulder and elbow but with severe referred forearm and hand pain. He refused to flex his fingers secondary to pain. A diagnosis of CRPS was made.

The patient was started on an aggressive physical therapy (PT) program. He had sessions every other day with routines at home facilitated by his mother. Six-week follow-up showed $100 \%$ improvement with normal range of motion and no edema or trophic changes. He was encouraged to return to daily activities while continuing with hand/wrist exercises.

\section{DISCUSSION}

Controversy surrounding the safety of and adverse reactions to vaccinations has always been an issue among health care providers and parents $(5,6)$. Despite the controversy, widespread immunizations have helped eliminate most childhood illnesses such as pertussis, diphtheria, polio, and measles. Unsubstantiated and hypothetical links to diseases such as autism, autoimmune diseases, and multiple sclerosis have fueled the benefitvs-risk arguments $(7,8)$. Not only are vaccines themselves being challenged, but the additives and preservatives in the vaccine preparations have contributed to questions of harm (9). However, most physicians agree that vaccination programs have contributed significantly to public health in disease prevention, and advocate childhood immunizations.

Despite the benefits of the recommended vaccination guidelines, adverse reactions cannot be ignored. Vaccine-related adverse events can be divided into 4 categories: local reactions (erythema, injection-site edema), systemic reactions (fever), allergic reactions (delayed hypersensitivity, arthrus reactions), and diseaseattributable events (encephalopathy, Bell's palsy) (10).

There have been documented cases of CRPS in children following immunizations with hepatitis B (HBVx) and rubella vaccines $(11,12)$. No link was found between the 2 vaccines and the proposed conclusion was that these cases were a result of injection trauma or a reaction to the vaccine constituents.

Historically, CRPS has been known by other taxonomy such as reflex sympathetic dystrophy (RSD) and causalgia, but was clarified by the International Association for the Study of Pain (IASP) to be CRPS type I, which is not associated with a nerve lesion, and CRPS type II, associated with a major nerve injury (1). Signs and symptoms include continuing and disproportionate pain, color and temperature changes, edema, and changes in skin turgor and nail growth. On examination patients describe burning, hyperesthesia, hyperalgesia, or allodynia of the affected site. Harden and Bruehl (13) have proposed that diagnosis be confirmed with at least one symptom in each category of sensory, vasomotor, sudomotor/edema, and motor/trophic changes. There are no definitive laboratories or radiographic diagnostic modalities; however, other diagnoses such as rheumatologic, neurologic, and orthopedic causes must be ruled out before a CRPS diagnosis can be made $(14,15)$.

There are a few marked differences between the adult and pediatric population concerning CRPS. In children, there is a preponderance of girls over boys and lower limbs over upper limbs. The severity of the symptoms significantly outweighs the inciting event and some patients may not even report any correlating injury $(3,16)$.

Our patient displayed all of the diagnostic criteria as outlined by the IASP in addition to the more explicit criteria as proposed by Harden and Bruehl (13), which 
were proposed to increase specificity and exclude false positives. He exhibited classical symptoms of allodynia, hyperalgesia, edema, skin changes, and decreased range of motion.

Pediatric CRPS is an underdiagnosed disorder due to limited clinical information, confounding presentations, and lack of experience with the condition among pediatric health care providers $(14,16)$. Patients may go an average of about 12 weeks without treatment, seeing multiple physicians from different specialties before a diagnosis of CRPS is made. The delay in diagnosis and management may have significant consequences on the treatment and resolution of the condition $(3,17)$. Fortunately, this patient was promptly referred to the pain center. PT was started immediately and he showed complete resolution of his symptoms.

The treatment goal for CRPS is restoration of functionality, which can be accomplished by PT, drugs, interventions, and psychotherapy $(2,18)$. Intense and regular PT seem to produce the most effective results with more rapid cure rates when the diagnosis and treatment are initiated early or within 3 months. Adjunctive medications such as anticonvulsants and antidepressants can facilitate participation and compliance with PT sessions. Noninvasive and invasive interventions such as transcutaneous electrical nerve stimulators (TENS) units, acupuncture, and sympathetic blocks have been shown to be valuable alternative modalities for analgesia. They are not however, considered the sole treatment for CRPS and are, again, helpful only to engage the patient in PT $(2,4,19)$. Complete resolution of symptoms is frequent in children and occurs after approximately 11 to 15 weeks of therapy. The success rate seems to be more a reflection of the willingness of children to participate in PT rather than a difference in the nature of the disease in children vs adults. Conversely, children have a higher incidence of relapse, in the magnitude of $20 \%$ to $50 \%$ (3). The evidence is conflicting as to the amenability of treatment in relapse cases.

Children seem to have a significant psychological component to their syndrome. Children with CRPS are commonly overachievers and may have multiple stressors in their life including family dysfunction, divorce, sexual abuse, and parental pressure. There is usually no evidence of major psychiatric disorders, but a stress-related component to the syndrome should not be overlooked (20). Treatments that account for behavioral and psychological factors and support the patient's psychosocial environment with counseling and teaching of pain and stress management skills can be an essential addition to therapy (4).

Previously, 4 cases of CRPS have been reported following HBVx and one case following a rubella vaccination. Similar to our patient, the symptoms in the other vaccine-related cases of CRPS occurred soon after the inciting event and no other causes could be found for the immense pain and dysfunction $(11,12)$. Public concern regarding vaccine safety is aimed not only at perceived vaccine-associated diseases (autism, multiple sclerosis, autoimmune disorders, etc.) but also at the hazards of the components, i.e., preservatives, stabilizers, adjuvants, and biological growth media $(8,9)$. Unfortunately, the components of the vaccines in the other case reports are unavailable; however, based on the dates of the other reported CRPS cases, we can deduce the possible components $(7,9)$. The vaccine given to our patient did not contain preservatives. Review of available manufacturers' dates does not seem to show a common constituent to all 3 vaccines, and injection trauma seems to be the only common factor across all instances.

\section{CONCLUSION}

Physicians need to be aware of this uncommon and potentially debilitating disorder that may arise following vaccinations. CRPS is underdiagnosed in pediatrics mainly due to inexperience and delay in recognition of the condition. A thorough history and physical exam are all that is needed to make the diagnosis. The key to successful resolution of symptoms is early diagnosis and initiation of PT. Delays may cause undue pain, extended lengths of treatment, requirements for more intense therapy, and less likelihood for complete functional restoration. Because there seems to be no common constituent to all 3 vaccines, the resultant CRPS may be purely due to injection-site trauma. We encourage physicians to be wary of the syndrome and its link to multiple vaccines in order to initiate prompt treatment. 


\section{REFERENCES}

1. Diekema DS. Responding to parental refusals of immunization of children. Pediatrics 2005; 115:1428-1431.

2. Maldonado YA. Current controversies in vaccination: Vaccine safety. JAMA 2002; 288:3155-3158.

3. Offit PA, Jew RK. Addressing parents' concerns: Do vaccines contain harmful preservatives, adjuvants, additives, or residuals? Pediatrics 2003; 112:1394-1397.

4. Mera KE, Hackley B. Childhood vaccines: How safe are they? Am J Nurs 2003; 103:79-88.

5. Eldred BE, Dean AJ, McGuire TM, Nash AL. Vaccine components and constituents: Responding to consumer concerns. Med J Aust 2006; 184:170-175.

6. Moylett EH, Hanson IC. Mechanistic actions of the risks and adverse events associated with vaccine administration. J Allergy Clin Immunol 2004; 114:1010-1020.

7. Harden RN, Bruehl SP. Diagnosis of complex regional pain syndrome: Signs, symptoms, and new empirically derived diagnostic criteria. Clin J Pain 2006; 22:415-419.

8. Harden RN, Swan M, King A, Costa B, Barthel J. Treatment of complex regional pain syndrome: Functional restoration. Clin J Pain 2006; 22:420-424

9. Low AK, Ward K, Wines AP. Pediatric complex regional pain syndrome. J Pediatr Orthop 2007; 27:567-572.

10. Wilder RT. Management of pediatric patients with complex regional pain syndrome. Clin J Pain 2006; 22:443-448

11. Jastaniah WA, Dobson S, Lugsdin JG, Petty RE. Complex regional pain syndrome after hepatitis B vaccine. J Pediatr 2003; 143:802804.

12. Genc H, Karagoz A, Saracoglu M, Sert E, Erdem HR. Complex regional pain syndrome type-I after rubella vaccine. Eur J Pain 2005; 9:517-520.

13. Harden RN, Bruehl S, Stanton-Hicks M, Wilson PR. Proposed new diagnostic criteria for complex regional pain syndrome. Pain Med 2007; 8:326-331.

14. Small E. Reflex sympathetic dystrophy: Reflections from a clinician. Adolesc Med State Art Rev 2007; 18:221-225.

15. Sherry DD, Malleson PN. The idiopathic musculoskeletal pain syndromes in childhood. Rheum Dis Clin North Am 2002; 28:669685.

16. Finniss DG, Murphy PM, Brooker C, Nicholas MK, Cousins MJ. Complex regional pain syndrome in children and adolescents. Eur J Pain 2006; 10:767-770.

17. Murray CS, Cohen A, Perkins T, Davidson JE, Sills JA. Morbidity in reflex sympathetic dystrophy. Arch Dis Child 2000; 82:231-233.

18. Sherry DD, Wallace CA, Kelley C, Kidder M, Sapp L. Short- and long-term outcomes of children with complex regional pain syndrome type I treated with exercise therapy. Clin J Pain 1999; $15: 218-223$

19. Mogilevsky M, Jänig W, Baron R, Harden RN. Complex regional pain syndrome-a multifaceted disorder requiring multidimensional care: Case study. J Pain 2007; 8:677-681.

20. Sherry DD, Weisman R. Psychologic aspects of childhood reflex neurovascular dystrophy. Pediatrics 1988; 81:572-578. 